\title{
ON IONOSPHERIC COMPOSITION MEASUREMENTS WITH AN R.F. IMPEDANCE PROBE
}

\author{
E. K. MILLER* and H. F. SCHULTE \\ High Altitude Engineering Laboratory, Department of Aerospace Engineering, \\ University of Michigan, Ann Arbor, Mich. 48104, U.S.A.
}

(Received 31 March 1969)

\begin{abstract}
Consideration is given to extending various methods used for determining electron number density in the ionosphere to ion composition measurements. A technique involving the impedance of an antenna operated in a frequency range on the order of the ion cyclotron frequency to the electron cyclotron frequency appears promising. Theoretical impedance results for some idealized combinations of ions as well as typical ion mixtures encountered in the ionosphere illustrate application of the technique. The advantages and limitations of the impedance probe are discussed.
\end{abstract}

\section{INTRODUCTION}

It has been long realized that the impedance variation with frequency of an antenna immersed in a plasma such as the ionosphere has the potential of yielding the plasma electron number density. Considerably less attention has been directed however, to the possibility of determining the masses and relative abundances of the various ions in the plasma from the antenna impedance. This problem was apparently first considered by Smith and Brice (1964) who investigated the influence of the ions upon the propagation of radio waves in the anisotropic ionospheric plasma. They suggested the use of either a passive or active swept-frequency satellite or rocket-borne antenna for this purpose.

Subsequently, a laboratory demonstration of this basic approach for ion spectroscopy has been reported on by Baldeschwieler (1968). The technique used by Baldeschwieler was one of finding the frquency behavior of a planar capacitor between whose plates was interposed the plasma to be analyzed and a known static magnetic field. The information giving the masses of the various ions is incorporated in the characteristic frequencies at which the capacitor has a low impedance. The characteristic frequencies lead to the ion masses through a consideration of the permittivity of the magnetoplasma between the capacitor plates.

A similar approach may be applied to analyzing the frequency dependence of the impedance of an antenna in the same magnetoplasma. In a recent article Blair (1968) has investigated this subject and concludes that theoretically at least the method has promising potential for ionospheric diagnostics. However, some experimental measurements intended to investigate this phenomenon which have been reported by Shawan and Gurnett (1967) are not encouraging. Their impedance results do not conform to the expectations of the theory. In this paper we will examine some aspects of the problem not discussed by Blair, and consider some of the limitations imposed by carrying out the actual experiment in the ionosphere which may invalidate the theoretical assumptions.

\section{FORMULATION}

The development of the tensor permittivity for an anisotropic plasma follows from Maxwell's equations together with the particle equations of motion, which for the usual case of interest involve electrons only. The ions are considered of infinite mass and therefore unaffected by the time changing fields in the plasma. The situation encountered is not

* Present address: M.B. Associates, San Ramon, California, U.S.A. 
basically different when the ion motion is a factor, but the tensor permittivity then involves the ion constituents in the plasma as well as the electrons. Without giving the details of the development, which are reported on by Smith and Brice (1964), the magnetoplasma permittivity may be expressed, for a $z$-directed static magnetic field and $\mathrm{e}^{i \omega t}$ time dependence, as

where

$$
\epsilon=\left[\begin{array}{rrr}
\epsilon_{1} & \epsilon^{\prime} & 0 \\
-\epsilon^{\prime} & \epsilon_{1} & 0 \\
0 & 0 & \epsilon_{3}
\end{array}\right],
$$

and

$$
\begin{gathered}
\epsilon_{1}=1-\frac{X_{e} U_{e}}{U_{e}^{2}-Y_{e}^{2}}-\sum_{i=1}^{\mathrm{I}} \frac{X_{i} U_{i}}{U_{i}^{2}-Y_{i}^{2}}, \\
\epsilon^{\prime}=\frac{-i X_{e} Y_{B}}{U_{e}^{2}-Y_{e}^{2}}+i \sum_{i=1}^{\mathrm{I}} \frac{X_{i} Y_{i}}{U_{i}^{2}-Y_{i}^{2}} \\
\epsilon_{3}=1-\frac{X_{e}}{U_{e}}-\sum_{i=1}^{\mathrm{I}} \frac{X_{i}}{U_{i}}
\end{gathered}
$$

$$
\begin{aligned}
X_{e}=\frac{\omega_{p e}^{2}}{\omega^{2}} ; & X_{i}=\frac{\omega_{p i}^{2}}{\omega^{2}}, \\
Y_{e}=\frac{\omega_{h e}}{\omega} ; & Y_{\imath}=\frac{\omega_{h i}}{\omega}, \\
U_{e}=1+v_{e} / i \omega ; & U_{i}=1+v_{i} / i \omega .
\end{aligned}
$$

In these equations, $\omega_{p}$ and $\omega_{h}$ are the radian plasma and cyclotron frequencies, $\nu$ is the collision frequency, with the subscripts $e$ and $i$ denoting the electrons and ions respectively and $\mathrm{i}$ is summed over the number of ion species present, denoted by $I$. Also,

$$
\begin{array}{cc}
\omega_{p e}^{2}=\frac{q^{2} N_{0}}{\epsilon_{0} m_{e}} ; & {\omega_{p i}{ }^{2}}^{2}=\frac{q^{2} A_{i} N_{0}}{\epsilon_{0} m_{i}}, \\
\omega_{h e}=\frac{q B_{0}}{m_{e}} ; & \omega_{h i}=\frac{q B_{0}}{m_{i}},
\end{array}
$$

with $B_{0}$ the static magnetic field strength, $\epsilon_{0}$ the permittivity of free space, $-q$ the electron charge, $N_{0}$ the static electron number density, and $m_{e}$ and $m_{i}$ the masses of the electron and $i$ th ion. The fractional abundance of the $i$ th ion is given by $A_{i}$, and we assume

$$
\sum_{i=1}^{\mathrm{I}} A_{i}=1,
$$

there being no multiply charged or negative ions present.

Maxwell's equations can now be written

$$
\begin{aligned}
\nabla X \mathbf{E} & =-i \omega \mu_{0} \mathbf{H} \\
\nabla X \mathbf{H} & =i \omega \epsilon_{0} \mathbf{\epsilon} \cdot \mathbf{E}
\end{aligned}
$$

with $\mathbf{E}$ and $\mathbf{H}$ the electric and magnetic fields and $\mu_{0}$ the permeability of free space. The development of the impedance of an antenna in the magnetoplasma from (3) and (4) is a 
very complex problem. The solution may be greatly facilitated, however, by assuming the source to be a specified current distribution. Contrary to the usual antenna problem, this technique eliminates the necessity of solving for the antenna current in the course of finding the antenna impedance. The approach generally gives good results for the antenna impedance if the antenna is short compared with the wavelengths of the excited fields. An additional assumption which further simplifies the theoretical treatment, is the quasi-static approximation, which is essentially a low frequency limit that requires the antenna-lengthto-wavelength ratio to approach zero; it is thus consistent with the current distribution assumption previously mentioned.

The theoretical development of the quasistatic formulas for the impedance of cylindrical and spherical antennas in a magnetoplasma has been given previously by Balmain (1964, 1966). We show the resulting formulas for the antenna impedance which have been used for the calculations to be discussed. They are, for the cylindrical antenna,

$$
Z_{i n}=\frac{1}{i 2 \pi \epsilon_{0} L} \cdot \frac{1}{\epsilon_{1} \omega \sqrt{F}}\left[\ln \left[\frac{L}{\rho} \frac{2 F}{(a+\sqrt{F})}\right]-1\right]
$$

where $2 L=$ antenna length,

$\rho=$ antenna radius,

$a^{2}=\epsilon_{1} / \epsilon_{3}$,

$F=\sin ^{2} \theta+\mathrm{a}^{2} \cos ^{2} \theta$,

$\theta=$ angle of antenna axis with respect to magnetic field

and for the spherical antenna,

$$
Z_{i n}=\frac{1}{j 8 \omega \pi \epsilon_{0}}\left\{\frac{1}{\epsilon_{1 s} m_{s}} \ln \left[\frac{1+m_{s}}{1-m_{s}}\right]\left[\frac{1}{R}-\frac{1}{R+S}\right]+\frac{1}{\epsilon_{1} m(R+S)} \ln \left(\frac{1+m}{1-m}\right)\right\}
$$

where $R=$ radius of sphere,

$m=\sqrt{1-\epsilon_{3} / \epsilon_{1}}=\sqrt{a^{2}-1}$,

$S=$ thickness of sheath.

In (7), a rough approximation for the ion sheath which forms about a body at floating potential in a warm plasma is included in the form of a concentric spherical shell of thickness $S$ from which electrons are excluded. The ions here are in the same concentration as in the plasma outside the sheath. Quantities denoted by a subscript $s$ in (7) are evaluated in the sheath, where we take $X_{e}$ to be zero. A more realistic sheath model which accounts for a radial variation in both the electron and ion densities may be readily put in the form of (7) by representing the sheath as a series of concentric spherical layers having slightly different electron and ion number densities. This approach has been used by Balmain et al. (1967) in comparing theoretical and experimental impedance results in the ionosphere for frequencies on the order of $f_{h e}$.

It should be re-emphasized that (6) and (7) are gross approximations to the situation encountered by an actual antenna in the ionosphere, ignoring as they do non-zero temperature effects, antenna motion relative to the medium, sheath asymmetries, plasma non-linearities, and the influence of the rocket or satellite carrier itself on the antenna behavior. In addition, the formulas above are for the steady-state situation, where an implicit assumption is that enough time has elapsed after turning on the antenna excitation at the frequency $\omega$ for the transients to die out and the antenna-plasma system to come to equilibrium. When the antenna is swept in frequency, the number of cycles that the excitation frequency is in the vicinity of the various characteristic frequencies of the plasma may 
be less than required to bring the plasma into equilibrium. The distance moved by the antenna during one oscillation period is also a factor. These considerations are not of crucial importance at the electron cyclotron frequencies $\left(f_{h_{e}}\right)$ encountered in the $E$-region of the ionosphere and above (typically on the order of $1 \mathrm{MHz}$ ), since the antenna, even at satellite velocities moves less than $1 \mathrm{~cm}$ during one cyclotron-frequency period. Also, a frequency sweep rate as high as $1 \mathrm{decade} / \mathrm{sec}$ means the frequency varies by less than 1 per cent from $f_{h_{e}}$ for almost $10^{4}$ cycles. When the ions are considered on the other hand, the cyclotron frequencies in the ionosphere may vary from 25 to $800 \mathrm{~Hz}$, so that the corresponding numbers may have extremes of $400 \mathrm{~m}$ and 0.2 cycles. This comparison serves to emphasize the differences between the ions and electrons as far as their contribution to the effective plasma permittivity is concerned. For the sake of brevity, the impedance probe technique under discussion will be referred to in the following as the ICIP, standing for 'Ion Composition Impedance Probe'.

\section{NUMERICAL EXAMPLES}

Allowing for the effect of a positive ion sheath around the antenna is more straightforward in the case of the spherical antenna than for the cylindrical antenna. Because of this, and also because the spherical antenna impedance has no field-orientation dependence as does the cylindrical antenna, thus simplifying the interpretation of the experimental results, most of our subsequent consideration will be devoted to the spherical antenna. In order to demonstrate the similarity of impedance results obtained with the two geometries however, the impedance of both antenna types is shown as a function of frequency in Fig. 1 for both a one-ion and two-ion plasma sheathless case, and the following parameter values:

$$
\begin{aligned}
L & =3.048 \mathrm{~m} \\
\rho & =0.01 \mathrm{~m} \\
R & =0.055 \mathrm{~m} \\
f_{p e} & =1 \mathrm{MHz} \\
f_{h e} & =1.47 \mathrm{MHz} \\
v_{c B} & =10^{4} \mathrm{sec}^{-1} ; \quad \theta=0^{\circ} .
\end{aligned}
$$

One ion plasma

$$
\begin{aligned}
& A_{1}=1 \\
& m_{1}=16 \mathrm{amu} \\
& v_{c 1}=10 \mathrm{sec}^{-1}
\end{aligned}
$$

Two ion plasma

$$
\begin{aligned}
& A_{1}=A_{2}=0.5 \\
& m_{1}=16 \\
& m_{2}=1 \\
& v_{c 1}=10 \mathrm{sec}^{-1} \\
& v_{c 2}=160 \mathrm{sec}^{-1} .
\end{aligned}
$$

The antenna radii and length have been chosen to conform to typical experimental parameters while $f_{p e}, f_{h e}$ and $v_{c e}$ are chosen as representative of the ionosphere at a height of $100-200 \mathrm{~km}$. The ion masses are for atomic oxygen and hydrogen, which are the principal ions in the altitude range $500-1000 \mathrm{~km}$, this ion combination being selected to illustrate the mass-separation dependence of the impedance. The ion collision frequencies are selected in the ratio used so that the ion cyclotron minima have similar shapes for the two ions. The question of the impedance dependence on ion mass, relative abundance and collision frequency will be considered separately below. 


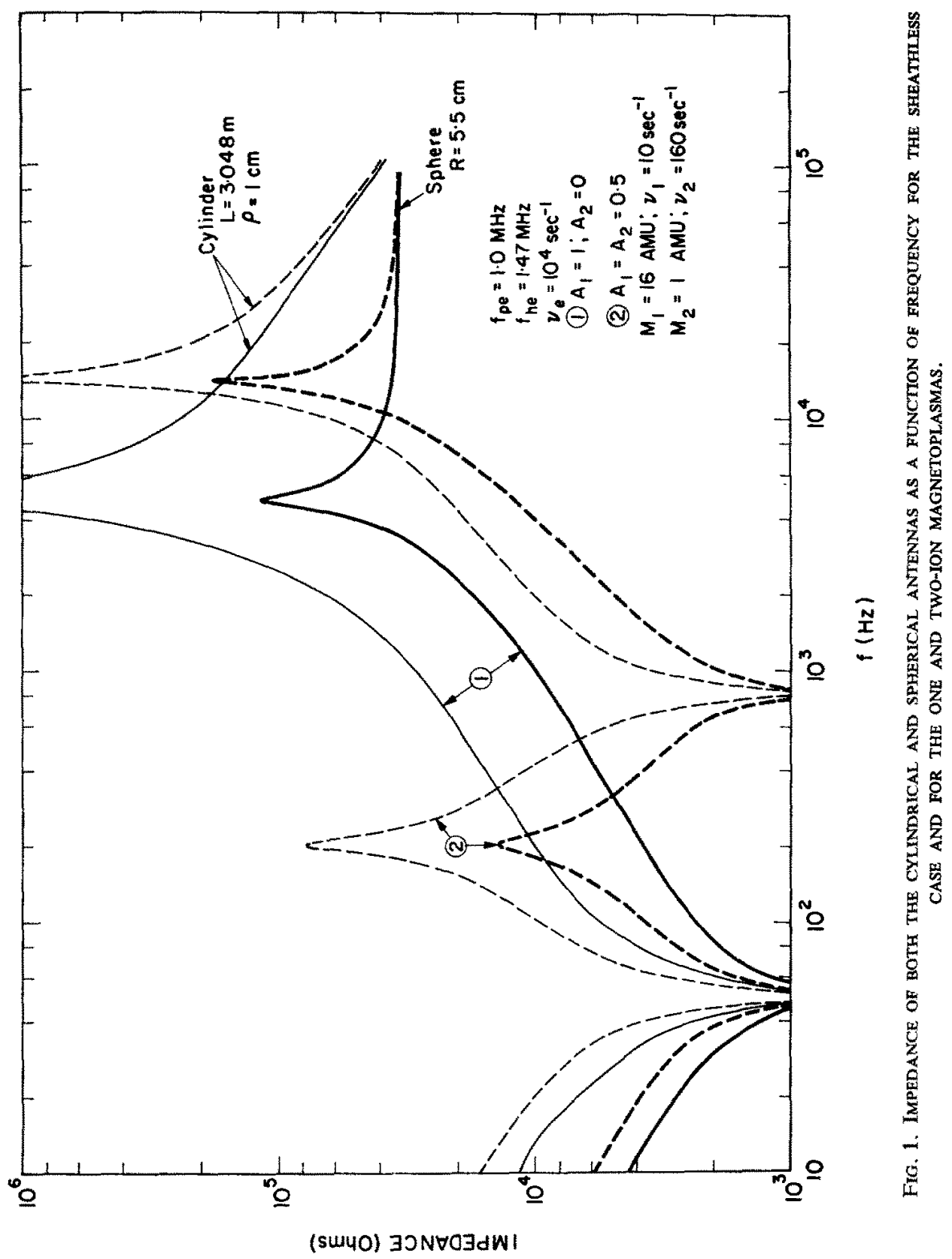


An examination of the impedance curves for the two antenna geometries shows that while the magnitudes are different, the location in frequency of their respective minima and maxima are the same, for both the one-ion and two-ion plasma. This is a demonstration that the minima and maxima frequencies result from the medium properties and not of the particular antenna geometry, as will be further verified below. The minima in impedance are related to the ion cyclotron frequencies while the maxima are hybrid resonances, the highest one shown involving the ion and electrons, and in the case of a multiple-ion plasma, the lower maxima involving ions only.

Before presenting additional impedance results for the spherical probe, it is worthwhile to investigate the resonance phenomena shown in Fig. 1, to arrive in particular at an understanding of the frequency dependence of the resonance upon ion mass and relative abundance. An examination of (10) and (11) shows that the impedance will become large when $\epsilon_{1} \rightarrow 0$, a situation usually referred to as a resonance, and small when $\epsilon_{1}$ is large, a condition denoted as a cutoff (Allis et al., 1963). If the collision frequencies are all zero, then $\epsilon_{1}$ can become exactly zero, so that impedance infinities would thus occur when

$$
1-\frac{X_{e}}{1-Y_{e}^{2}}-\sum_{i=1}^{\mathrm{I}} \frac{X_{i}}{1-Y_{i}^{2}}=0 .
$$

Impedance zeros would result when

$$
\frac{1}{1-\frac{X_{e}}{1-Y_{e}^{2}}-\sum_{i=1}^{I} \frac{X_{i}}{1-Y_{i}^{2}}}=0 .
$$

It is easy to see that impedance zeros occur whenever

$$
Y_{e}=1 \quad \text { or } \quad Y_{\imath}=1
$$

i.e. at the various ion and electron cyclotron frequencies. If the collision frequencies are non-zero, impedance minima occur at frequencies where $\epsilon_{1}$ has maxima. For small $v / \omega$ values, these impedance minima are not appreciably shifted from the $Y_{e}$ or $Y_{i}=1$ values. The effect of a variable collision frequency will not be considered further, except to remark that as the collision frequency increases, the impedance extrema tend to decrease in amplitude. Impedance zeros or infinities are of course not physically possible, the nonlinear plasma behavior limiting the field magnitudes in the cutoff and resonance regions.

The cutoff frequencies, or the impedance zeros (or minima), are thus seen to be determined by the respective cyclotron frequencies of the various ions and the electrons as shown by Fig. 1. Since the cyclotron frequency is mass dependent only and not a function of the particle number density, we see that the cyclotron cutoff frequencies serve to identify the masses of the constituent plasma ions. The resonance frequencies, which are solutions to (8a), are not so simply stated. Since they may involve more than one plasma constituent or characteristic frequency, they are denoted as hybrid resonances. An cxact solution for these hybrid frequencies when several ions are present is very complex analytically. If only a two-ion plasma is considered and some reasonable approximations are made, the hybrid resonance frequencies may be easily obtained however. Since these hybrid resonanance frequencies have been derived by Smith and Brice, we present only their results here. The highest resonance frequency involves only the electrons alone, and is the well-known upper hybrid frequency $f_{t}$, given by

$$
f_{t}^{2}=f_{h e}^{2}+f_{p e}^{2}
$$


Another hybrid resonance $f_{i e}$ can occur between the ion plasma frequencies and electron plasma frequency, and if we denote the ion to electron mass ratio by $M_{i}$, then

$$
f_{i e}{ }^{2}=\frac{f_{p e}^{2} f_{h e}^{2}}{f_{t}^{2}} \sum \frac{A_{i}}{M_{i}} .
$$

The lowest hybrid resonance occurs in the vicinity of the ion cyclotron frequencies, and for two ions we have for the ion-ion hybrid resonance $f_{j}$.

$$
f_{i}{ }^{2}=\frac{f_{h e}{ }^{2}}{M_{1} M_{2}}\left[\frac{A_{1} M_{1}+A_{2} M_{2}}{A_{1} M_{2}+A_{2} M_{1}}\right] .
$$

The subscript $j$ will be used to number the ion-ion resonances, in order of increasing magnitude, there being $I-1$ resonances for the $I$-ion plasma. Since $I=2$ in this example, $j=1$ only.

An examination of the resonance frequencies (10) and the cutoff frequencies (9) reveals that a cutoff occurs between each resonance, i.e. the impedance maxima are separated by impedance minima. The minima identify the masses of the various plasma constituents, while the maxima contain information about the relative abundances. For example, having determined $M_{1}$ and $M_{2}$ from (9), (10c) may be used to find the ratio of $A_{1}$ to $A_{2}, f_{h e}$ being obtained from a knowledge of the Earth's magnetic field. Application of (10b) then leads to a relationship between $f_{p e}$ and $A_{1}$ for example, so that a separate measurement of $f_{p e}$ (possibly from a relaxation resonance) will then determine $A_{1}$. While $f_{p e}$ must be known for an absolute determination of ion number density, the relative abundances may be found from ion hybrid resonances and

$$
\sum_{i=1}^{\mathrm{I}} A_{\imath}=1
$$

Consequently, (10b) could then be used to find $f_{p e}$ from the known $A_{i}$ and $M_{i}$. Thus, another method is available for finding the electron number density. Since the ion-electron hybrid resonance frequency is sheath dependent whereas the ion-ion hybrid resonances are not (for the sheath model assumed here), a comparison between $f_{p e}$ obtained from (10b) with a relaxation resonance measurement may provide information of value about the sheath thickness and consequently, the electron temperature.

While the discussion above has been confined to the two ion plasma, the same principles apply to the $I$-ion plasma. The $I-1$ ion-ion hybrid resonance frequencies, together with (3) and (10b) provide necessary information for converting the $I$ relative ion abundances into absolute number densities. The most attractive feature of using this impedance probe technique for measuring the ion concentrations is that it is the frequencies at which the various resonances and cutoffs occur (which are properties of the medium) rather than the absolute antenna impedance, that provides the composition information. The measurement is thus potentially less subject to error and theoretical misinterpretation since the frequency can be generally measured more accurately than the impedance. In addition the cutoff and resonance frequencies may more reasonably be expected to be correctly predicted by the theory than the absolute antenna impedance.

It is natural to ask at this point what sort of accuracy is required in the frequency measurement to obtain a desired accuracy in the relative ion abundances. In addition, it is pertinent to inquire about the sensitivity of the various resonance frequencies to changing relative ion abundances, since (10b) and (10c) both contain a dependence on the ion mass and concentration. Consequently, we present in Figs. 2 and 3 respectively the ion-electron 
hybrid resonance frequency, $f_{i \varepsilon}$ and the ion-ion resonance frequency $f=1$ as a function of relative ion abundance $A_{1}\left(A_{2}=1-A_{1}\right)$ for $f_{p e}=1.47 \mathrm{MHz}, f_{h e}=1.47 \mathrm{MHz}$ and various ion mass ratios, with the mass $M_{1}$ being 32 amu corresponding to molecular oxygen. We see that the ion-electron hybrid resonance is on the order of $10 \mathrm{kHz}$, or about $10^{-2} f_{p e}$, while the ion-ion hybrid resonance is on the order of $100 \mathrm{~Hz}$. The sensitivity of the resonances to changing $A_{1}$ is seen to be very dependent on the ratio $M_{1} / M_{2}$, and for $M_{1} / M_{2} \leq 2\left(M_{1} / M_{2}=\right.$ $32 / 30$ corresponds to $\mathrm{O}_{2}{ }^{+}$and $\left.\mathrm{NO}^{+}\right)$, a very large change in relative abundance produces a

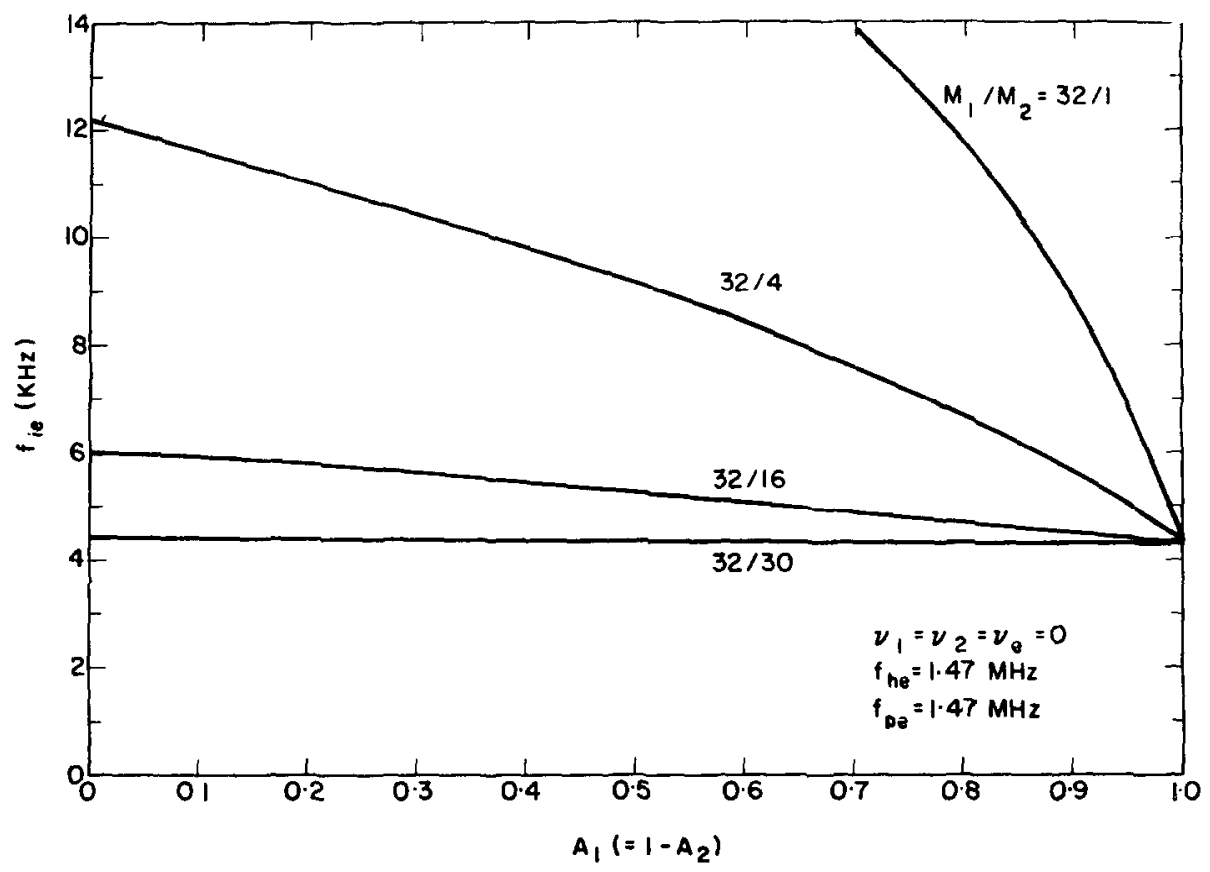

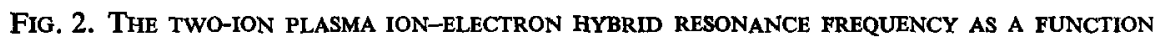
OF RELATIVE ION ABUNDANCE WITH THE ION MASS Ratio a PaRAMETER.

very small change in the resonance frequency. If frequency changes of 5 per cent are accurately detectable, then relative abundance changes will be measureable only for $M_{1} / M_{2}$ ratios on the order of 2 or larger, where the fractional changes in $A_{1}$ on the order of 10 per cent or less may be seen from the ion-ion resonance. It should again be noted that the ion-electron hybrid resonance frequency is not required to obtain the relative ion abundances, but it is useful in establishing their absolute concentrations. Since its location is proportional to the electron density, its relative insensitivity to changing ion composition is not too important.

Our discussion of the impedance probe has thus far been confined to the impedance magnitude, rather than considering for example, the resistive and reactive impedance components separately, i.e. the impedance phase. Since the antenna reactance changes sign as the frequency passes through each resonance or cutoff for small collision frequencies, the antenna reactance would alone be sufficient to determine these frequencies. However, the impedance magnitude is often more readily measured than the separate components, so that from an experimental standpoint it may be preferable to deal with the impedance magnitude. Nonetheless, this certainly does not rule out the desirability of attempting to 


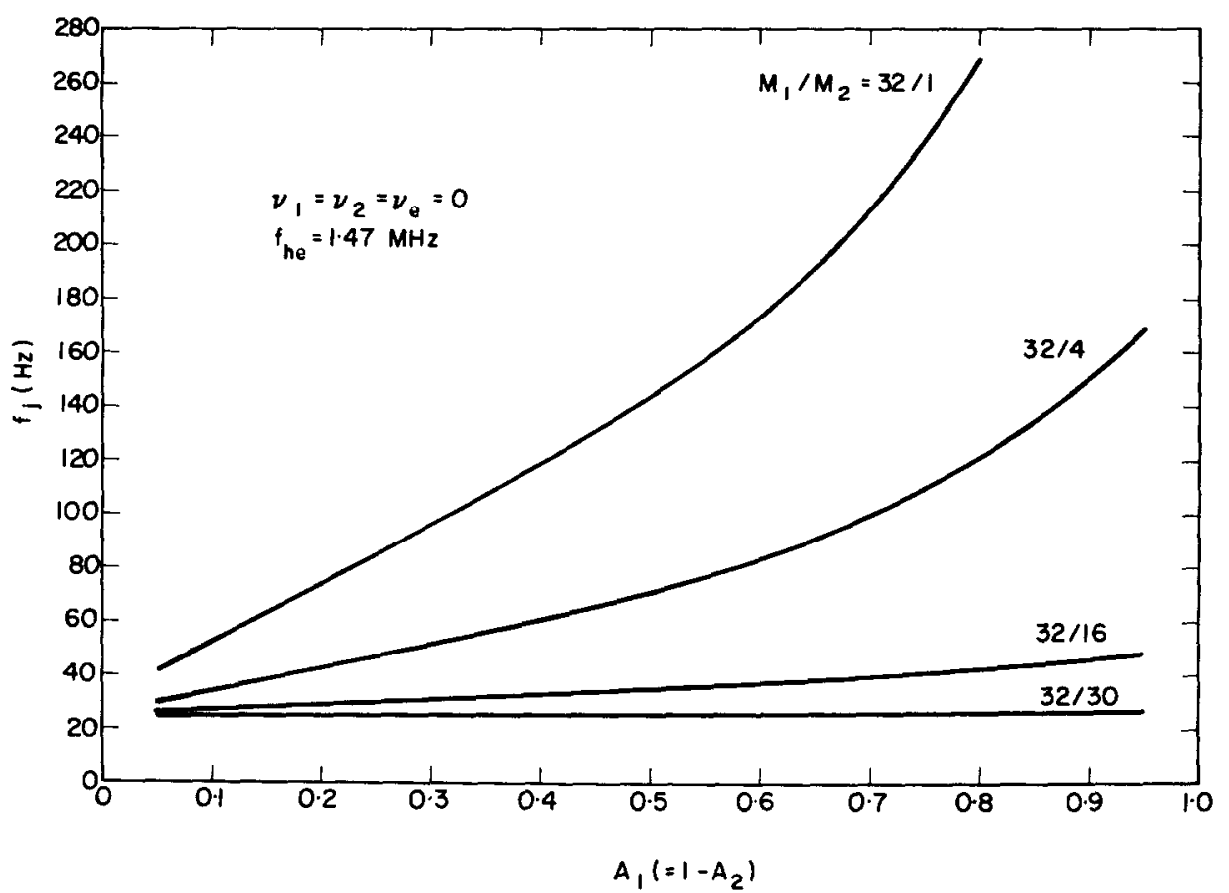

Fig. 3. The TWO-ION PLASMa ION-ION HYBRID RESONANCE FREQUENCY AS A FUNCTION OF RELATIVE ION ABUNDANCE WITH THE ION MASS RATIO A PARAMETER.

measure the impedance phase, as well as its magnitude, since the additional information resulting from this may prove very fruitful. For example, the reactance zero shifts away from the resonance and cutoff frequencies for sufficiently large collision frequencies, so that the possibility exists of using this property to also obtain the corresponding collision frequencies. In this preliminary study however, we will confine the results to the impedance magnitude alone.

With these considerations behind us, it is now worthwhile to present some impedance curves which illustrate the role of the other parameters, such as the sheath thickness, as well as to show graphically the impedance behavior with changing ion abundance. In Fig. 4 is shown the spherical antenna impedance as a function of exciting frequency for the sheathless case, with the relative abundance of the two ions of 16 and 1 amu (corresponding to $\mathrm{O}^{+}$ and $\mathrm{H}^{+}$ions) a parameter. The impedance curves on this graph show rather strikingly the shift in location of the ion-ion resonance with changing ion abundance. It is especially interesting to see that the depth of the impedance minimum at the ion cyclotron cutoff decreases with decreasing density of the particular ion. The dependence of the resonance frequencies upon the ion mass ratios and abundance is seen to agree with that predicted by $(10 \mathrm{~b})$ and $(10 \mathrm{c})$, where an increased proportion of the heavier ion is shown to shift the ion-electron resonance downward in frequency, while the ion-ion resonance is shifted upward.

Curves corresponding to those of Fig. 4 are shown in Fig. 5 for the case of a 5 electron Db length $\left(D_{l}\right)$ thick ion sheath with

$$
\begin{aligned}
D_{l} & =\sqrt{\left(k T_{e} \epsilon_{0} / N\right)} / q, \\
S & =\mathrm{R}+5 D_{l},
\end{aligned}
$$




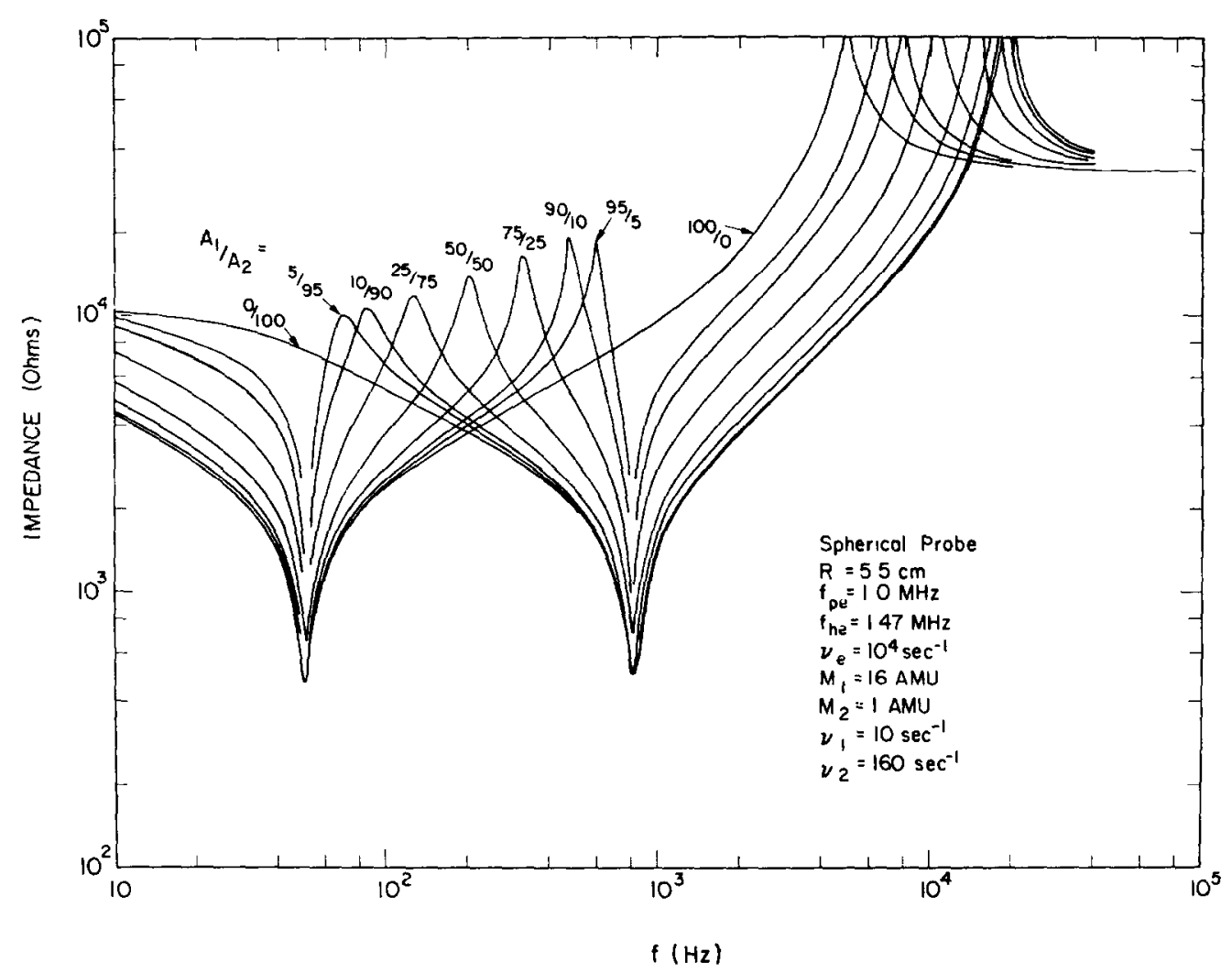

Fig. 4. IMPEdance OF THE SPHERICAL PROBE IN THE TWO-ION PLASMA FOR THE SHEATHLESS CASE AS A FUNCTION OF FREQUENCY WITH THE RELATIVE ION ABUNDANCE RATIO A PARAMETER.

where $k$ is Boltzmann's constant and an electron temperature $T_{e}$ of $1500^{\circ} \mathrm{K}$ has been used in the calculations. The impedance is increased one to two orders of magnitude compared with the sheathless case, and the ion-electron resonance frequency is shifted upward compared with the sheathless case. The ion-ion resonances occur at the same frequencies for both the sheathed and sheathless cases, however, as do the ion cutoff frequencies.

The curves of Figs. 4 and 5, are quite striking and appear to indicate that the ICIP may be promising for ionospheric diagnostics. It must be emphasized however, that the plasma parameters used are idealized as far as the ion mass ratios and collision frequencies of the real ionosphere are concerned. Thus in order to demonstrate the results which may be more typical of the actual ionosphere, we present in Figs. 6 and 7 the antenna impedance vs. frequency curves calculated from actual measured ion composition profiles in the ionosphere.

The results of Fig. 6 are shown over the altitude range $100-300 \mathrm{~km}$ at $50 \mathrm{~km}$ intervals using the ion composition measurements of Johnson, Meadows and Holmes (1958) taken from a rocket shot 20 November, 1956, together with the ion and electron collision frequencies* and electron densities for a 'hot' ionosphere given by Chapman (1956). The

* It is unrealistic, as pointed out by a reviewer, to use an abundance-independent ion collision frequency. An examination of the ion collision frequency values used to obtain the curves of Fig. 6 however, leads to the conclusion that the results would not be significantly changed by using an abundance-dependent ion collision frequency. The qualitative implications of Fig. 6 are therefore not invalidated by the use of the same collision frequency value for all three ions. 


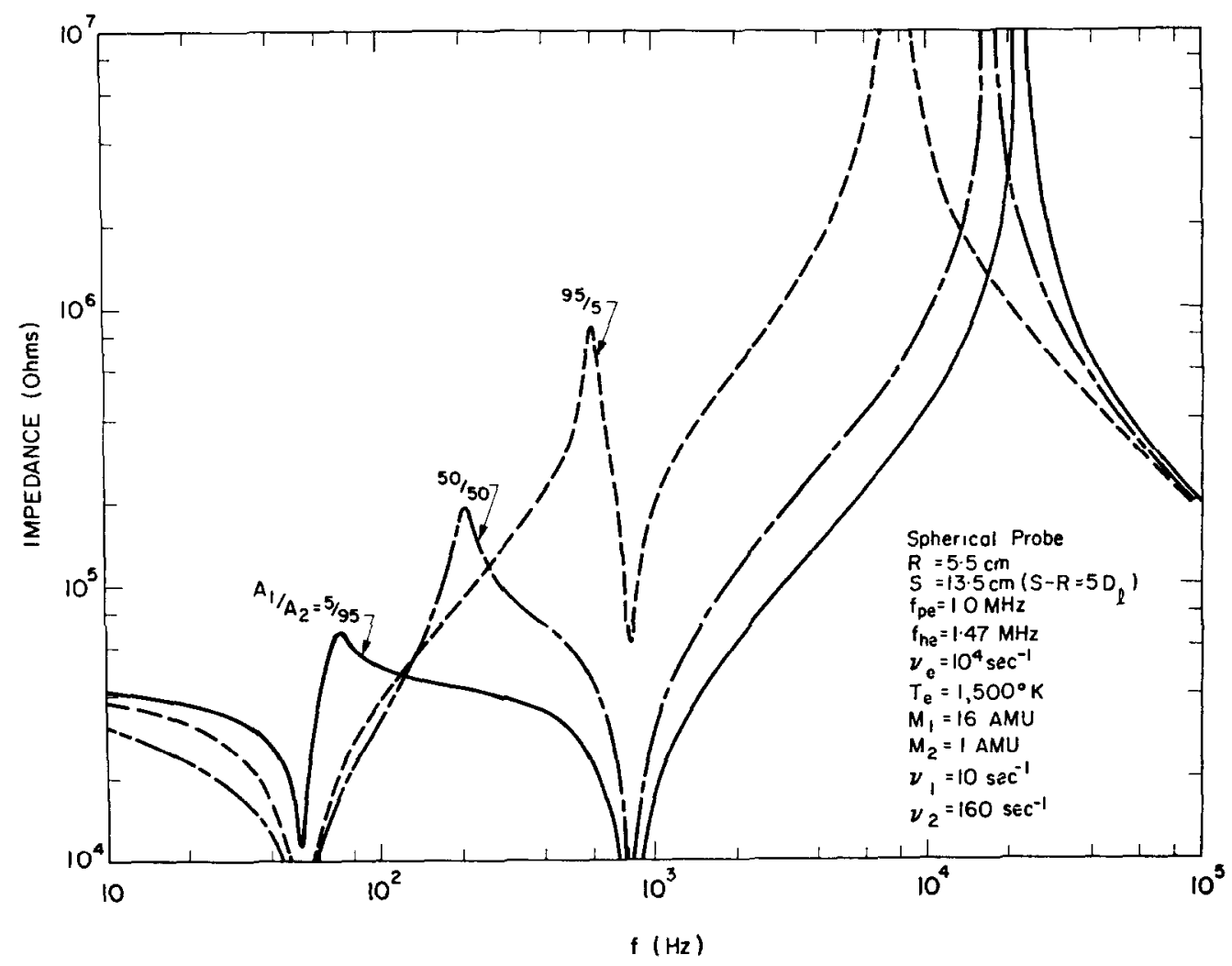

FIG. 5. IMPEDANCE OF THE SPHERICAL PROBE IN THE TWO-ION PLASMA FOR THE $5 D_{l}$ ION SHEATH AS A FUNCTION OF FREQUENCY, WITH THE RELATIVE ION ABUNDANCE RATIO A PARAMETER.

numerical values used for these parameters are shown on the graph. The curve for $100 \mathrm{~km}$ altitude is seen to be very flat, and except for frequencies greater than about $100 \mathrm{kHz}$, almost independent of frequency. This impedance behavior is caused by the relatively large collision frequencies for this height in the ionosphere, which as may be deduced from the impedance formula given by (7), has the effect of cancelling the $1 / \omega$ term in the impedance over a relatively large frequency range.

At an altitude of $150 \mathrm{~km}$, the collision frequencies are considerably smaller than at 100 $\mathrm{km}$ and the impedance begins to show the frequency dependence previously found for the more idealized plasma models. It should be emphasized however that there are three ions present in the plasma, but because of the still comparatively high collision frequency, only one broad minimum is seen in the impedance. Proceeding upward another 50 to $200 \mathrm{~km}$, we see that two minima now occur, one at about $50 \mathrm{~Hz}$, corresponding to $\mathrm{O}^{+}$, and the other at about $26 \mathrm{~Hz}$, which appears to be the result of the separate minima for $\mathrm{NO}^{+}$and $\mathrm{O}_{2}^{+}$ coalescing due to the still appreciable collision frequencies and the closeness of their respective masses. Finally at $250 \mathrm{~km}$ altitude three impedance minima are seen corresponding to the three ions present in the model ionosphere which has been used. Since the $\mathrm{NO}^{+}$ and $\mathrm{O}_{2}{ }^{+}$ions are now present with an abundance for each of about 7 per cent of the total ion population, their cyclotron cutoffs and the ion-ion resonance between are not very pronounced. The $300 \mathrm{~km}$ curve is for a one-ion plasma, consisting of $\mathrm{O}^{+}$alone, and thus 


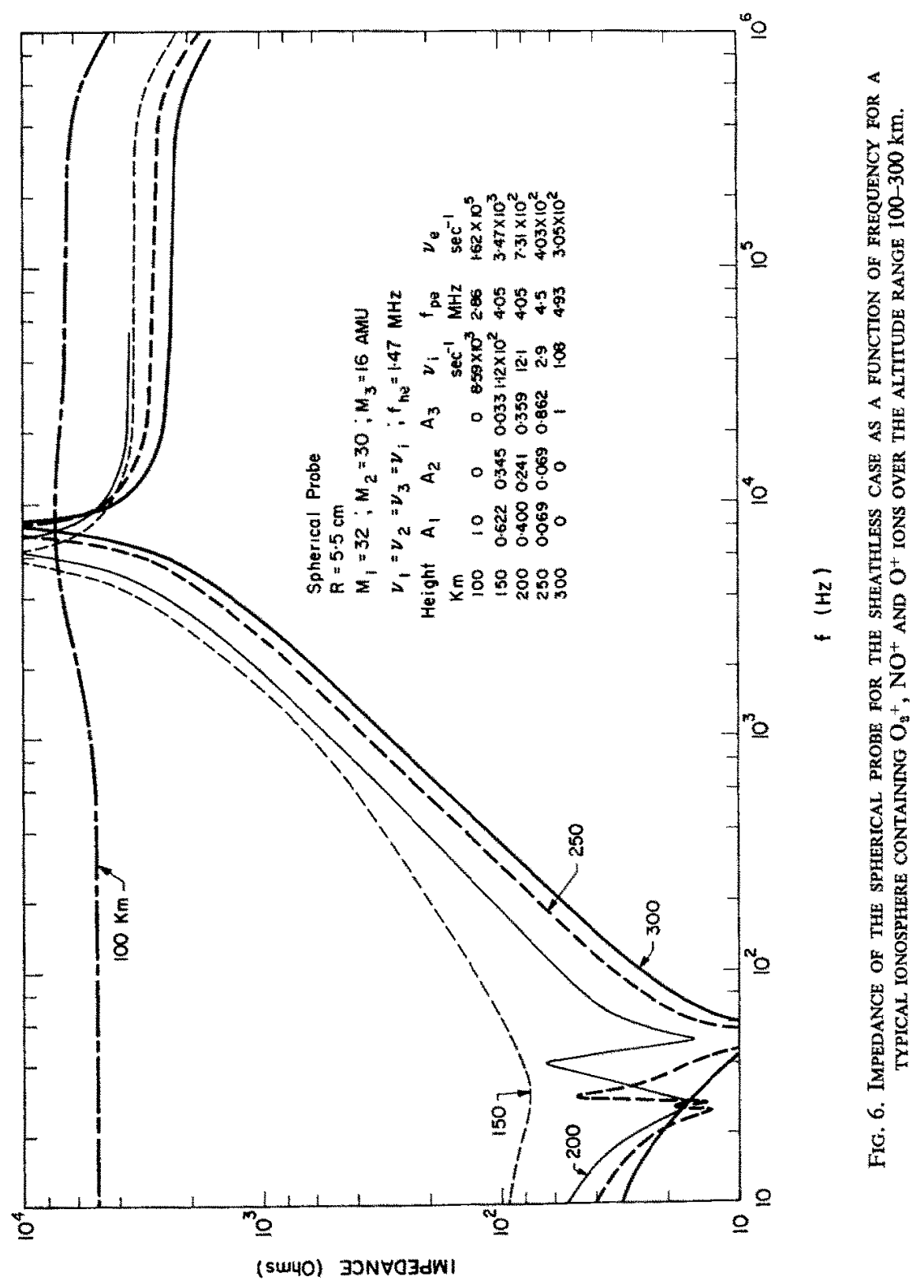



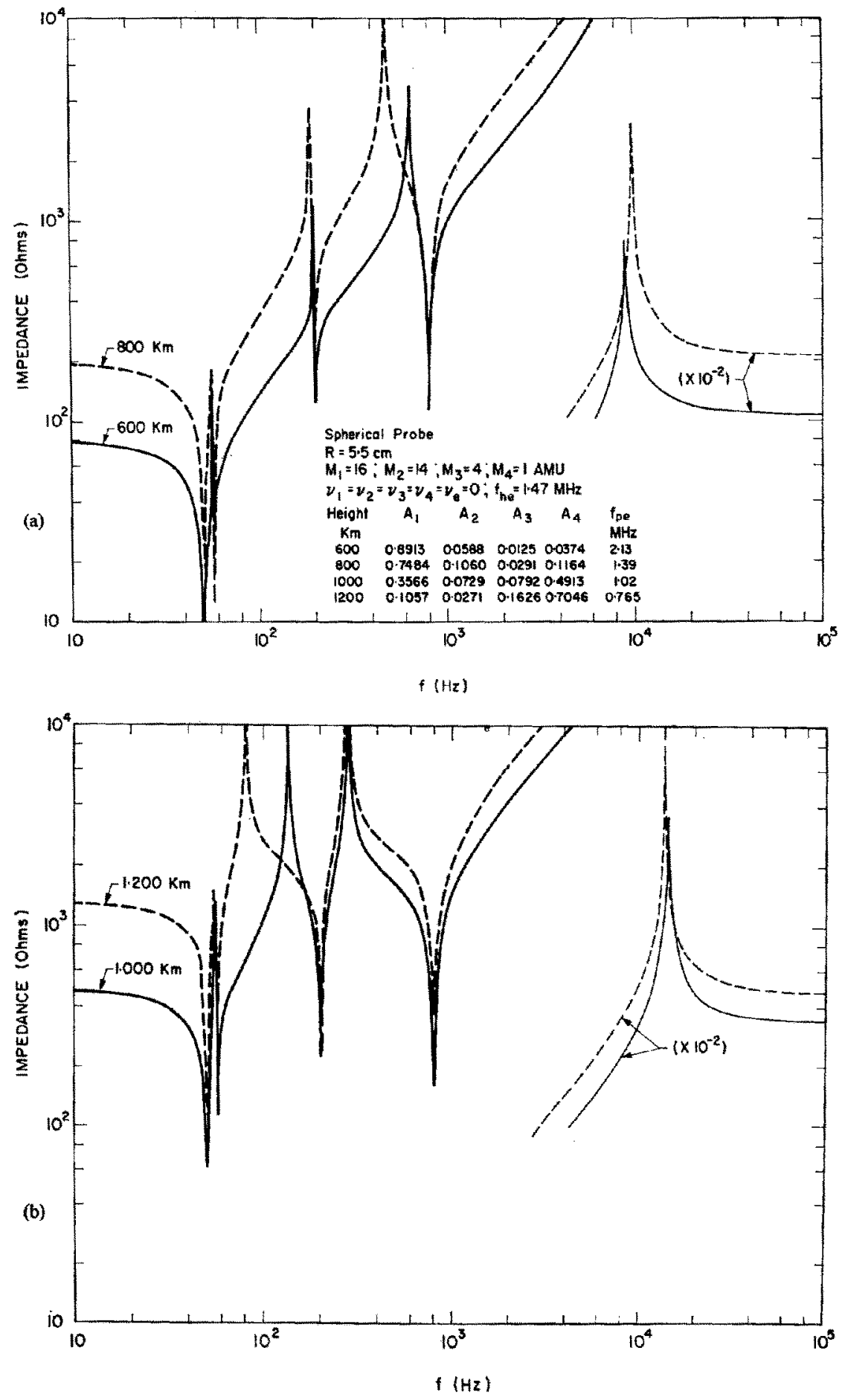

FIG. 7. IMPEDANCE OF THE SPHERICAL PROBE FOR THE SHEATHLESS CASE AS A FUNCTION OF FREQUENCY FOR A TYPICAL TONOSPHERE CONTAINING $\mathrm{O}^{+}, \mathrm{N}^{+}, \mathrm{He}_{2}{ }^{+}$AND $\mathrm{H}^{+}$IONS FOR THE ALTTTUDES: (a) 600 AND $800 \mathrm{~km}$; AND (b) 1000 AND $1200 \mathrm{~km}$. 
has one minimum at the $\mathrm{O}^{+}$cyclotron frequency. In each of the curves from $150 \mathrm{~km}$ and up, there is seen to be an upward shift in the ion-electron resonance, which is caused mainly by the increasing electron density with altitude. For two of the curves however, at 150 and $200 \mathrm{~km}$, the electron density is the same, the upward shift here resulting from the increased relative abundance of the lighter mass ion.

Figure 7 shows the probe impedance vs. frequency curves for an altituderange of $600-1200$ $\mathrm{km}$ at $200 \mathrm{~km}$ intervals. The ion and electron density values are taken from the results summarized by Ghosh (1967). Since the ion and electron collision frequencies are negligible in this altitude range, they have been set equal to zero for purposes of the calculations. The four curves are shown on two separate graphs for the sake of clarity, since due to the use of zero collision frequency values the minima and maxima are quite sharp and may overlap. For convenience, a constant value for $f_{h e}$ of $1.47 \mathrm{MHz}$ has been used for these calculations so that the shifts in the ion-ion resonance would be due to changes in fractional abundances only. In practice, the magnetic field would decrease significantly in the altitude range considered here. The change of magnetic field with increasing altitude could easily be taken into account in the reduction of experimental data.

The curves of Fig. 7 show that cyclotron cutoffs occur for each ion in spite of the fact that the fractional abundance of a particular ion may be as low as 1 per cent. It thus appears that identification of the less abundant ions present in the ionosphere would be feasible, although their relative concentrations may be difficult to obtain accurately because of the small shifts in the various ion-ion resonances which result from fairly substantial changes in the relative abundances. The ion-ion resonances are seen to shift more when located between the cyclotron cutoffs of ions whose masses differ by the larger amounts, a feature mentioned in the previous discussion.

Our discussion of the antenna impedance has not considered the possibility of the probe interacting with the plasma in ways other than the exciting of an electromagnetic field which is modified by the effective permittivity of the surrounding plasma. In a somewhat related study, Aggson and Kapetankos (1966) considered the source impedance of an antenna in the ionosphere which interacts with the plasma by drawing both an ion and electron current, as well as a photoemission current. Their numerical results for a cylindrical antenna $100 \mathrm{~cm}$ in length and $1 \mathrm{~cm}$ in dia. moving with satellite velocity indicate that the impedance is primarily resistive for frequencies below $100 \mathrm{kHz}$ and altitude of $200-1000 \mathrm{~km}$ in the daytime, the night-time resistive domain occurring for somewhat lower frequencies. Above these frequencies the impedance becomes primarily capacitive. Over the 200-1000 $\mathrm{km}$ altitude range, the antenna impedance may vary by more than an order of magnitude, being as low as $10^{4} \Omega$ in the daytime, with the night-time value roughly an order of magnitude larger. Because the minimum impedance value obtained by Aggson and Kapentankos is on the order of the largest impedance values found in Figs. 6 and 7 where the appropriate ionospheric parameter values have been used, it appears that the influence of charge collection from the plasma by the antenna on the impedance would be small, since the two impedances would be in parallel connection. This comparison is not wholly valid because Aggson and Kapetankos used a cylindrical geometry in their calculations, while we have used a spherical geometry. In any case, the effect of charge collection and photo-emission by the antenna may be largely minimized by coating the antenna with a suitable dielectric material.

Another important consideration is the applicability of the sheath description used for these calculations. We have already noted the increased impedance which results when the 
antenna is surrounded by an ion sheath a few electron Debye lengths thick, because of the decreased sheath conductivity when ions only are present in the sheath. If the ions too do not contribute to the sheath conductivity, perhaps due to the motion of the antenna through the plasma so that the ions move through the sheath without appreciably interacting with the r.f. fields there, then as shown by the first term of (7a) with $\epsilon_{1 s} \sim 1, Z_{\text {an }} \sim 10^{8} \Omega$ at $f=10^{2} \mathrm{~Hz}$. This large value of impedance would effectively screen the probe from the effect of the plasma, contained in the second term of (11a), so that the frequency dependence of the probe would be $f^{-1}$ over the frequency range of interest here. A behavior of this sort is reported in a recent experiment conducted by Shawhan and Gurnett (1967). This possibility suggests that it may be desirable to decrease the free-space probe impedance by d.c. biasing the antenna to reduce the series sheath component. An attractive alternative may be a passive, noise-monitoring probe to look for noise enhancement at the various characteristic frequencies, an approach suggested by Smith and Brice (1964).

\section{CONCLUSION}

This investigation has considered the possibility of measuring ion composition in an ionospheric-type plasma by methods other than the spectrometric techniques currently in use. A motivation for this work has been the desire to obtain an ion composition measurement scheme that is inherently much less complex and less prone to calibration error than are current spectrometers designed for use in the ionosphere.

Consideration of various techniques that are used for electron density measurements and the feasibility of extending them to ion composition determination has led to the impedance probe as potentially the most promising method to pursue. Based on the quasistatic formulation of Balmain for the zero-temperature magnetoplasma, the ICIP is found to exhibit minima and maxima in impedance as a function of frequency. The impedance minima supply information about the relative masses of the various constituents ions, reducing to absolute ion masses if the ionospheric magnetic field is known. From the impedance maxima are obtained the relative fractional abundances of the various ions, as well as potential information concerning the electron density, electron temperature and sheath thickness.

The most attractive feature of the ICIP is that it is the frequencies at which the impedance minima and maxima occur, rather than the absolute impedance magnitude, which supplies the ion composition. A frequency measurement is generally more easily performed to a given accuracy than an impcdance measurcment, while in addition, the various charactcristic frequencies are dependent on the plasma medium and likely to be less subject to theoretical uncertainties than is the absolute antenna impedance.

The theoretical approach used is certainly limited in sophistication, neglecting as it does, non-zero temperature effects, probe velocity through the medium, and possible non-linear plasma behavior. Consequently the results obtained in this study, while intriguing and quite promising, cannot be accepted without some caution. It appears that further theoretical effort to bring the actual physics and assumed model closer together would be extremely difficult because of computational problems. It would be reasonable however, to seriously consider carrying out an experiment as part of a rocket payload. Some experimental results could be very informative in demonstrating the feasibility of the technique and determining the desirability of further theoretical effort. 


\section{REFERENCES}

Aggson, T. L. and Kapetankos, C. A. (1966). On the impedance of a satellite borne VLF electric field antenna. Goddard Space Flight Center, Greenbelt, Md., Rep. No. X-612-66-380.

Allis, W. P., Buchsbaum, S. J. and Bers, A. (1963). Waves in Anisotropic Plasmas. M.I.T. Cambridge, Mass.

Ament, W. S., KATZIN, J. C., Katzin, M. and Koo, B. Y.-C. (1964). Impedance of a cylindrical dipole having a sinusoidal current distribution in a homogeneous anistropic ionosphere. Rad. Sci. J. Res. 379-405.

BACHYNSKI, M. P. (1966). Sources in unbounded plasmas. Invited Rev. Paper, 15th Gen. Ass. URSI, Munich, Germany; RCA Victor Res. Rep. No. 7-801-50.

BALDESCHWIELER, J. D. (1968). Ion cyclotron-resonance spectroscopy. Science 159, 263-273.

Balmain, K. G. (1964a, 1964b). The impedance of a short dipole antenna in a magnetoplasma. Univ. of Illinois, Urbana, Ill., Aeron. Rep. No. 2; IEEE Trans. Antennas Propag. 12, 605-617.

BaLmain, K. G. (1966). Impedance of a spherical probe in a magnetoplasma. IEEE Trans. Antennas Propag. 14, 402-403.

BALMAIN, K. G., OKSIUTIK, G. and FeJeR, J. (1967). R.F. probe admittance in the ionosphere: comparison of theory and experiment. Spring URSI Mtg, Ottawa, Ontario, Canada.

BLAIR, W. E. (1968). Ionospheric diagnostics using resonance of an electric dipole impedance. Radio Sci. 3, 155-161.

Brice, N. M. (1965). Ion effects observed in radio wave propagation in the ionosphere. URSI Symp. Electromagnetic Wave Theory, Delft, Netherlands.

Chapman, S. (1956). The electrical conductivity of the ionosphere: a review. Nuovo Cim. Suppl. 4, 13851412.

Chu, C. M., LaRue, J. and van Hulsteyn, D. B. (1966). Investigations on excitation and propagation in ionized media. Rome Air Dev. Ctr, Rep. No. RADC-TR-65-484.

Dougherty, J. P. and MonaGhan, J. J. (1966). Theory of resonances observed in ionograms taken by sounders above the ionosphere. Proc. $R$. Soc. A289, 214-234.

Fejer, J. A. and CALVERT, W. (1964). Resonance effects of electrostatic oscillations in the ionosphere. J. geophy. Res, 69, 5049-5062.

GALEJS, J. (1966a). Impedance of a finite insulated cylindrical antenna in a cold plasma with a longitudinal magnetic field. IEEE Trans. Antennas Propag. 14, 727-736.

GALEJS, J. (1966b). Impedance of a finite, insulated antenna in a cold plasma with a perpendicular magnetic field. IEEE Trans. Antennas Propag. 14, 737-748.

Grosн, S. N. (1967). Ionospheric characteristics from altitude variations of positive ion densities. Univ. of Michigan Scient. Rep. No. 05627-9-S.

HeRman, J. R. (1964). A method for determining $D$-region electron-density profiles utilizing a capacitive impedance rocket probe. J. geophys. Res. 69, 2329-2336.

Johnson, C. Y., Mradows, E. B. and Holmes, J. C. (1958). Ion composition of the arctic ionosphere. J. geophys. Res. 63, 443-444.

MeYER, P. (1967). Impedance of a sphere in a magnetoplasma. IEEE Trans. Antennas Propag. 15, 331-332.

MiLler, E. K. (1967a). Admittance of the infinite cylindrical antenna in a lossy plasma II. The incompressible magnetoplasma. Univ. of Michigan Scient. Rep. No. 05627-11-S.

PyaTI, V. P. (1966). Determination of capacitance in anisotropic media. IEEE Trans. Antennas Propag. 14, 803.

Schultz, F. V. and GrofF, R. W. (1966). The radiation produced by an arbitrarily oriented dipole in an infinite, homogeneous, warm, anisotropic plasma. Purdue Univ. Tech. Rep. No. EE66-22.

SESHADRI, S. R. (1965a). Radiation resistance of a linear current filament in a simple anisotiopic medium. IEEE Trans. Antennas Progag. 13, 819-820.

SESHADRI, S. R. (1965b). Radiation resistance of elementary electric current sources in a magnetoionic medium. Proc. IEE 112, 1856-1868.

SESHADRI, S. R. (1966a). Radiation from a current strip in a uniaxially anistropic plasma medium. Can.J. Phys. 44, 207-217.

SESHADRI, S. R. (1966b). Effect of insulation on the radiation resistance of an electric dipole in a simple anistropic medium. Proc. IEE 113, 593-600.

Shawhan, S. D. and Gurnett, D. A. (1967). Preliminary results from the Javelin 8. 45 VLF rocket experiment. Univ. of Iowa Preliminary Rep. No. 67-60.

SMITH, R. L. and BRICE, N. (1964). Propagation in multicomponent plasmas. J. geophys. Res. 69, 50295040.

Tosprer, A. J. and Comisar, G. G. (1966). Observations of a resonance probe effect near the ion plasma frequency. Presented at the Fall Mtg Div. Plasma Phys. American Physical Society, Boston, Mass. 